\title{
The sandwich effect reassessed: Effects of streaming, distraction, and modality
}

\author{
ALASTAIR P. NICHOLLS and DYLAN M. JONES \\ Cardiff University, Cardiff, Wales
}

\begin{abstract}
The degree of disruption from interleaving auditory irrelevant items within a sequence of tobe-remembereditems - the sandwich effect - was examined in two experiments. Previous demonstrations of the effecthave shown that the penalty for interleaving items is small and that changing irrelevanttokens is no more damaging than repeating ones (contrary to the classic changing state effect of irrelevant sound). The results of Experiment 1 suggest that these earlier results were due to the lack of tokens in the irrelevant sequence (in part, the result of using a span method). The results of Experiment 2 also show that the sandwich effect was marked and, further, that it comprised two elements, one due to the partitioning of relevant from irrelevant streams (which may be promoted by similarity of identity within sequence or pitch disparity between sequences), and the other a classic irrelevant sound effect (with effects of changing state). The results are discussed in terms of the role of perceptual organization within and between modalities in short-term memory.
\end{abstract}

If, for immediate serial recall of spoken lists, irrelevant verbal items of one class (e.g., letters) are interpolated between the to-be-remembered verbal items of another class (e.g., digits), there is an increase in errors of serial recall resulting from the presence of the irrelevant events; this is known as the sandwich effect (e.g., Hitch, 1975). All recorded instances of the sandwich effect have shown it to be reliable but small in magnitude. From one standpoint, the small magnitude of the effect is not surprising in view of the well-developed capacity of the auditory system of streaming sequences according to timing (e.g., Bregman, 1990), but from another standpoint, there should be a marked detrimental effect due to the mere presence of irrelevant sound, quite independent of any streaming effects. This last, irrelevant sound effect, has been demonstrated many times, invariably accompanied by the changing state effect - that is, a changing sequence of irrelevant items is more disruptive than a repeated sequence (e.g., Jones \& Macken, 1993, 1995a, 1995b, 1995c; Jones, Madden, \& Miles, 1992; LeCompte, 1994, 1995). However, most of the demonstrations of the irrelevant sound effect have been with visually presented to-be-remembered lists (e.g., Colle \& Welsh, 1976; Jones \& Macken, 1993; Jones et al., 1992;

A.P.N. received support from the Biotechnology and Biological Sciences Research Council (U.K.) in the form of a research studentship and from the Thomas Wilson Educational Trust (U.K.). Thanks are due Amelia Woodward for help in data collection. Earlier versions of the manuscript benefited greatly from suggestions by Bill Macken, Rob Houghton, Robert Hughes, and Sebastien Tremblay. D. M. Jones is also Adjunct Professor at the Department of Psychology, University of Western Australia. Correspondence should be addressed to D. M. Jones, School of Psychology, Cardiff University, P. O. Box 901, Cardiff CF10 3YG, Wales (e-mail: jonesdm@cardiff.ac.uk).

-Accepted by previous editorial team
LeCompte, 1995, 1996; Martin-Loeches \& Sommer, 1998; Salamé \& Baddeley, 1982). In the handful of studies using auditory to-be-remembered stimuli, not only is the sandwich effect small, and necessarily the irrelevant sound effect also small, but in no case was there a changing state effect (Baddeley, Papagno, \& Andrade, 1993, Experiment 5; Hamilton \& Hockey, 1974, Experiments 2 and 3; LeCompte, 1996, Experiment 3).

What evidence there is suggests, therefore, that the nature of the irrelevant sound effect seems to be differentboth in magnitude and character-when presented interleaved with auditory to-be-remembered events, and the purpose of the present study was to find out why this is so. One possibility is that the processing of auditory lists is functionally different from that of visual lists (a suggestion made by a number of researchers; e.g., Baddeley, 1986; Hanley \& Broadbent, 1987; LeCompte, 1996; Neath, 1999, 2000 ), and this in some way modulates the interaction with the irrelevant sound effect. Another possibility is that there are small procedural differences that might account for the effect. For example, in the study of LeCompte (1996, Experiment 3) keyboard responses were required in contrast to the more common written recall (cf. Miles, Jones, \& Madden, 1991; Penney \& Blackwood, 1989). Hamilton and Hockey (1974, Experiments 2 and 3) hinted at an effect of change (no formal analysis was undertaken directly between changing and repeated conditions) but permitted participants to adopt a free recall response strategy, as opposed to the more usual serial recall in irrelevant sound studies.

The focus of the present paper is on one set of experiments in which the method of measuring memory is likely to have militated against a strong sandwich effect. Baddeley et al. (1993) used a span method: Participants were given three trials with lists beginning at two digits and increasing by one every third trial, span being calculated as "the 
mean number of digits in the last three sequences correctly recalled" (Baddeley et al., 1993, p. 863). Performance was compared between a control condition (to-be-remembered digits presented in the absence of irrelevant material) and various sandwich conditions, in which a single irrelevant item (single sandwich) was presented before and after each to-be-remembered digit. Baddeley et al. (1993, Experiment 5), although finding a sandwich effect of modest size, found no difference in span between repeated (repeated letter) and changing conditions (letters selected from the alphabet). One possibility is that, as a side effect of the span method adopted by Baddeley et al. (1993), the number of irrelevant items presented to participants was small, making the deliberate selective processing of the to-beremembered sequence a relatively simple task; at the same time, the number of irrelevant items was small compared with that in a typical irrelevant sound experiment. Both factors are likely to have reduced the likelihood of obtaining effects. In the study of Baddeley et al. (1993), digit-span was about five items, which means that for those conditions in which a sandwich was present, only six irrelevant items were presented in all. An established feature of the irrelevant sound effect is that the degree of disruption is proportional to the number of tokens in the irrelevant sequence: the token-dose effect (Bridges \& Jones, 1996). It seems possible that in the Baddeley et al. (1993) study, the dose was too small to show either an irrelevant sound effect or a changing state effect.

As a starting point for the reassessment of the sandwich effect, the present paper begins with an attempt to replicate the finding of Baddeley et al. (1993, Experiment 5), with the speculation in mind that their results were a consequence of their adopting a design in which a single irrelevant item was sandwiched between items in a span task. In Experiment 1, we examined the effect of increasing the dose of interpolated irrelevant material (from one to two interpolated items). Increasing the number of irrelevant items in this way should have two effects: First, it should make the partitioning of relevant from irrelevant streams more facile, on the grounds that auditory streaming is more effective in sequences with higher rates of presentation (see, e.g., Nicholls \& Jones, 2002); second, the changing state irrelevant sound effect should become more evident.

\section{EXPERIMENT 1}

In Experiment 1, we attempted to replicate the procedure of Baddeley et al. (1993, Experiment 5), while extending the conditions to the case in which the number of irrelevant tokens was increased. Performance under repeated and changing irrelevant items was assessed with the use of a span-type measure. The method was identical to that of Baddeley et al. (1993, Experiment 5), with two exceptions. First, in addition to the single-sandwich condition (one interpolated irrelevant item), the present experiment investigated the effect of interpolating two irrelevant items between to-be-remembered items (double sandwich), allowing the interplay between the factors of dose (single or double sandwich) and sequence type (repeated or chang- ing) to be examined. Second, whereas Baddeley et al. (1993) compared performance in a repeated sandwich condition with two changing conditions-one with a predictable sequence and the other with an unpredictable sequence-we compared repeated sequence of items with only one changing (unpredictable) condition. Typically, there is no effect of predictability (e.g., Jones et al., 1992), and no theory supposes that there should be one.

\section{Method}

Participants. Thirty participants from Cardiff University took part in the study in exchange for financial reward. All participants were native English speakers who reported normal hearing.

Apparatus and Materials. To-be-remembered items were chosen from the digits 1-9, and the irrelevant items were the letters A through $\mathrm{H}$. A female speaker recorded all items in monotone (with a fundamental frequency of approximately $210 \mathrm{~Hz}$ ). Items were sampled with a 16-bit resolution, at a sampling rate of $48 \mathrm{KHz}$ using Sound Designer II software (Digidesign, 1994). All items were compressed digitally to a duration of $250 \mathrm{msec}$ with the use of the same software, without adversely affecting the auditory attributes of the stimulus, such as pitch.

For all conditions, the to-be-remembered digits were presented at a rate of one per second. Digits within sequences were ordered quasi-randomly with the precaution that consecutive numbers should not follow a familiar pattern (e.g., 2, 4, 6. .) and that a digit should not be followed by another that was one more or less than it. Four different types of interpolated sequence were prepared in which the factors of dose (single and double) and sequence type (repeated and changing) were jointly manipulated. That is, any sandwich trial consisted of either one or two items (single or double sandwich) between to-be-remembered items. At the same time, the interpolated material was composed of either a repeated-item or a changing-item sequence. In all sandwich conditions, one irrelevant item was presented before the first to-be-remembered item of a sequence and one after the last (i.e., each sequence began and finished with a distractor stimulus).

As in the study of Baddeley et al. (1993), irrelevant items in the single-sandwich conditions were presented at a rate of one per second. The onset of each to-be-remembered item followed the offset of an irrelevant item by $250 \mathrm{msec}$. Similarly, the offset of each to-beremembered item was followed $250 \mathrm{msec}$ later by the onset of an irrelevant item. In the double-sandwich sequences, two irrelevant items were presented every second. The onset of each to-be-remembered item followed the offset of an irrelevant item by approximately $83 \mathrm{msec}$. The offset of each to-be-remembered item was followed $83 \mathrm{msec}$ later by the onset of an irrelevant item. The two irrelevant items interpolated between to-be-remembered items were separated (offset to onset) by approximately $83 \mathrm{msec}$.

The repeated irrelevant sequences comprised the repetition of the letter A. For the changing conditions, irrelevant items were chosen from the letters A through $\mathrm{H}$ and were presented in a nonpredictable order.

Design. Five conditions were compared in a repeated-measures design: a control condition (in which to-be-remembered items were presented without irrelevant material), and four sandwich conditions, two with repeated irrelevant tokens, and two with changing irrelevant tokens. The type of sandwich sequence was manipulated jointly with dose, in which either one (repeated/single and changing/ single) or two (repeated/double and changing/double) irrelevant items were interpolated between to-be-remembered items. A Latin square counterbalanced the order of the five conditions. Six participants were tested in each of the five orders.

Procedure. All participants were tested individually in a soundproof laboratory. Sequences were presented through headphones set to a comfortable level [corresponding roughly to $60 \mathrm{~dB}(\mathrm{~A})$ ]. 
The experiment commenced with sequences two digits in length. Three consecutive trials were presented at this length under each of the five conditions before the length of the digit sequence was increased by one. The procedure was continued until sequences of length nine were completed, regardless of the errors made in the intervening trials. The participants were instructed to remember the sequence of digits, while ignoring any letters that might be presented in the same trial. After the sequence was presented, the participants were cued to recall in strict serial order on a response blank. The participants initiated the onset of each trial. Prior to the experiment proper, the participants completed two practice trials from each condition. With their consent, the participants' compliance with the procedure was monitored through a closed-circuit television.

\section{Results and Discussion}

In line with the scoring procedure adopted by Baddeley et al. (1993), digit-span was defined as the mean length of the last three sequences recalled correctly before the occurrence of an error.

Figure 1 illustrates digit-span under all conditions. Span was substantially smaller when irrelevant sequences were interpolated between the to-be-remembered sequences. The results replicate the findings of Baddeley et al. (1993, Experiment 5), in that the changing state effect was not present in a single-sandwich condition, but there was a changing state effect in the double-sandwich condition.

When digit-span in the five conditions was compared in a one-way repeated-measures analysis of variance (ANOVA), the main effect of condition was significant $[F(4,116)=$ $\left.30.19, M S_{\mathrm{e}}=0.930, p<.0001\right]$. A Tukey/Kramer comparison $(\alpha=.05$; critical difference for all comparisons $=$ .69) demonstrated that span was not significantly different in the two repeated conditions (span: repeated/ single = 4.7 ; repeated/double $=4.6$ ). Although the difference between repeated/double and changing/double $($ span $=3.3)$ was significantly different, the difference between repeated/single and changing/single ( $\operatorname{span}=4.3$ ) was not reliable. Further, span in the changing/double condition was significantly inferior to span in the changing/single condition. Lastly, digit-span in the control condition ( $\operatorname{span}=6.0$ ) was significantly greater than it was in any of the sandwich conditions.

In order to summarize economically the interaction between the changing nature of the interpolated material and the number of irrelevant items interpolated between to-beremembered items, a $2 \times 2$ repeated-measures ANOVA was undertaken, from which the control condition was excluded, involving the factors sequence type (repeated vs. changing) and dose (single vs. double). The analysis revealed a significant main effect of sequence type $[F(1,29)=$ $\left.35.83, M S_{\mathrm{e}}=0.66, p<.0001\right]$ and dose $[F(1,29)=$ $\left.35.83, M S_{\mathrm{e}}=0.65, p<.01\right]$. Importantly, the interaction term proved significant $\left[F(1,29)=8.24, M S_{\mathrm{e}}=0.72, p<\right.$ $.01]$. Clearly, the interaction arose due to the role of dose in determining span in the changing, but not in the repeated, sequences.

Experiment 1 supports the suggestion that the span task adopted by Baddeley et al. (1993) was insensitive to the effect of interpolated material owing to the relatively small number of irrelevant interpolated tokens. Indeed, by increasing the token dose in this way, we demonstrated in Experiment 1 that the sandwich effect is not, as Baddeley et al. (1993) suggested, modest and limited in magnitude. The presence of irrelevant material in a to-be-remembered list reduced span by between 1.3 and 2.7 digits compared with the reduction of one digit that was often found by Baddeley et al. (1993).

The size of the effect may be judged in relation to the Cohen's $d$ statistic. Typically, a $d$ of 0.2 is associated with a small effect, a $d$ of 0.5 with a medium effect, and a $d$ of 0.8 with a large effect. When calculated for Experiment 1 , it is clear that at its maximum, the sandwich effect is large. If we take the contrast between control and changing/ double, this yields a $d$ of 1.59 , clearly a large effect. With

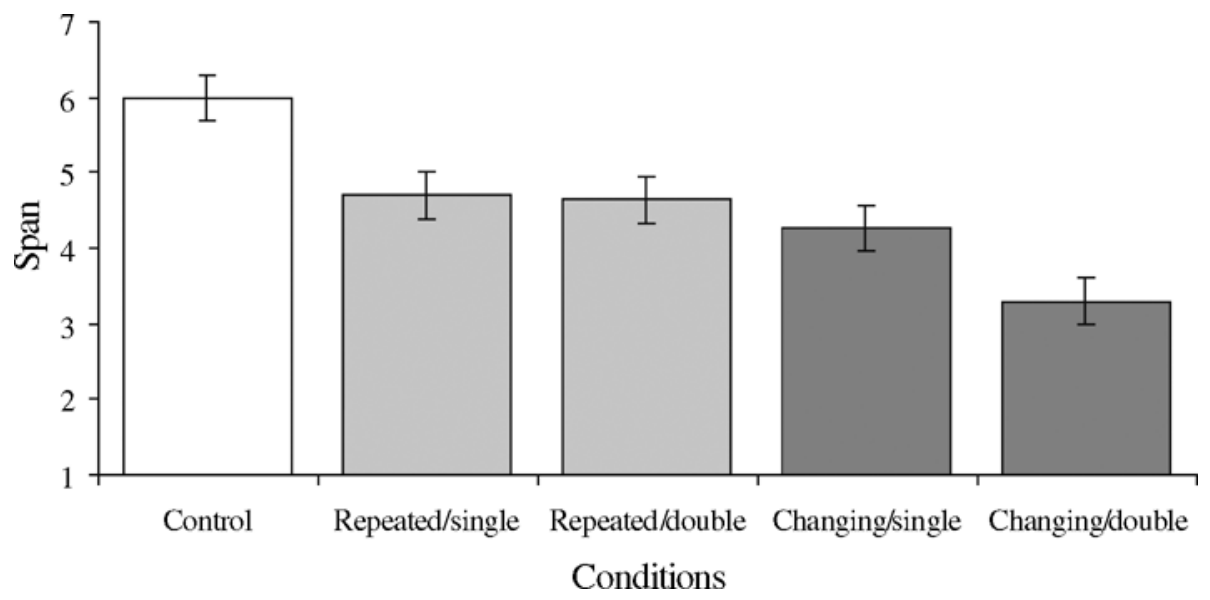

Figure 1. The effect of interpolated spoken irrelevant material on mean memory span (from Experiment 1). The changing nature of the irrelevant material (either repeated or changing) was manipulated jointly with dose: Either one (single) or two (double) irrelevant items were presented between to-be-remembered items. Standard error bars are displayed. 
the higher dose (the double sandwich), the changing state effect is also marked (repeated/double vs. changing/double; $d=0.78$ ). The contrast more closely akin to the conditions of Baddeley et al. (1993) - the repeated/single versus changing/single - produced a weak effect size $(d=0.24)$.

In Experiment 2, we examined in more detail how streaming interacts with the irrelevant sound effects in auditory lists. Two factors known to promote partitioning of auditory streams were covaried: The irrelevant sequence type (changing and repeated) was manipulated jointly with pitch difference (same as list pitch vs. five semitones below list pitch). The action of these factors on an auditory list was compared with a visual analogue. We argue that the effects of irrelevant sound on an auditory list when streaming is maximized should resemble irrelevant speech effects with a visually presented list (for which, necessarily, no comparable streaming is required). This should allow a comparison of the action of irrelevant sound on lists in the auditory and visual modality.

\section{EXPERIMENT 2}

Experiment 2 was composed of two parts, identical in every sense except for the presentation modality of the tobe-remembered list: In Experiment 2A, it was auditory, in Experiment 2B, visual. There were two major predictions for Experiment 2A. First, just as in Experiment 1, the degree of interference should in part be dictated by the ease with which the irrelevant sequence could be segregated perceptually from the to-be-remembered sequence. In Experiment $2 \mathrm{~A}$, two principles of streaming were exploited: similarity $\times$ identity (contrasting repeated and changing sequences, as in Experiment 1), and similarity of pitch between the irrelevant and the changing sequence. Second, there should be an irrelevant speech effect-that is, an effect of the mere presence of irrelevant items, so that repeated irrelevant items would impair serial recall less than changing irrelevant items. However, this differential effect of changing versus repeated sequences should be less noticeable when the changing irrelevant items were distinct in pitch from the to-be-remembered sequence.

The largest disruption of recall should be evident under conditions in which the irrelevant sequence was not only changing but also presented at the same pitch as the to-beremembered list. Under such circumstances, performance should be affected adversely from a changing state effect of irrelevant sound and, additionally, from the difficulty in streaming (neither repetition nor pitch difference was present here). Indeed, it was expected that recall should be improved (albeit not to the level of the repeated condition) when a changing irrelevant sequence was presented at a distinct pitch from the to-be-remembered list. That is, although a pitch difference should facilitate processes acting to partition relevant from irrelevant automatically, the changing nature of the irrelevant sequence should still invoke a changing state effect.

The results should take the form of an interaction: When the irrelevant sequence was made of repeated items, the effect of pitch should be small, but when the irrelevant sequence was changing, performance should generally be more impaired, but the impairment would be less when the pitch difference was large.

In Experiment 2B, the presentation of the to-beremembered items was visual but the items and the irrelevant auditory stimuli were identical to those used in Experiment $2 \mathrm{~A}$. The predictions were different in two respects: First, the effect of irrelevant items should be smaller generally, because of the difficulty of streaming relevant from irrelevant would not be evident, but, particularly, there would be a proportionately smaller effect of repeated items (the changing state effect) since the difficulty of partitioning would be reduced. Clearly, in Experiment 2B, there should be no interaction between the pitch of and the change within the irrelevant sequence.

\section{Method}

Participants. Fifty-two undergraduate students from the school of psychology at Cardiff University participated in Experiment 2 in return for course credit. All participants were native English speakers who reported normal hearing. None had participated in Experiment 1 . Twenty-six participants took part in Experiment $2 \mathrm{~A}$ and a different set of 26 participants took part in Experiment 2B.

Apparatus and Materials. The same timing between relevant and irrelevant items used in the double-sandwich conditions of Experiment 1 was adopted here. To-be-remembered items were chosen from the digits 1-9; irrelevant items were the letters A through $\mathrm{H}$. To-be-remembered sequences consisted of eight items presented at a rate of one per second. Digits were arranged within sequences with the same precautions as adopted in Experiment 1. In the auditory version (Experiment 2A), a female speaker recorded all stimuli in monotone (with a fundamental frequency of approximately $210 \mathrm{~Hz}$ ). Items were sampled with a 16-bit resolution, at a sampling rate of $48 \mathrm{kHz}$ with the use of Sound Designer II software (Digidesign, 1994). Further, all items were digitally compressed to a duration of $250 \mathrm{msec}$ using the same software.

In the visual version (Experiment 2B), each to-be-remembered item was presented for $250 \mathrm{msec}$ in a 72 -point Times New Roman font at the center of the monitor positioned in front of the participants. The materials in the visual version were presented using PsyScope software (Cohen, MacWhinney, Flatt, \& Provost, 1993).

In Experiments $2 \mathrm{~A}$ and $2 \mathrm{~B}$, the same four types of interpolated sequence were used, in which a repeated or changing irrelevant sequence was presented either at the pitch of the auditory to-beremembered list, or at five semitones below that used in Experiment 2A. Sound Designer II software (Digidesign, 1994) was used to transform irrelevant item pitch without changing the duration of the stimuli.

Irrelevant items in the repeated sandwich conditions comprised the repetition of the letter A. For the changing conditions, the letters A through $\mathrm{H}$ were presented in alphabetical order twice in a single trial. The sequence reverted back to the letter $\mathrm{A}$ after the presentation of $\mathrm{H}$ (i.e., . . F, G, H, A, B, C, etc.). Successive changing trials started with a different letter, but each time, the irrelevant sequence proceeded from this starting letter in alphabetical order.

Design. Five conditions were compared in a repeated-measures design: a control condition, in which only to-be-remembered items were presented, two repeated sandwich conditions (denoted by the letter R), and two changing sandwich conditions (denoted by the letter C). Within each type of sandwich condition, irrelevant items were presented either at to-be-remembered list-pitch (R0 and C0 conditions), or at five semitones below list-pitch (R5 and C5 conditions). Over the course of 80 trials, the participants completed 16 trials for 
each condition. Trials were ordered in a quasi-random fashion so that the same condition was not presented more than two times in successive trials. The same order of conditions was presented to all participants. The design was identical to that of Experiment 2B.

Procedure. The same procedure adopted in Experiment 1 was used in Experiment 2, with the following exceptions. The participants were forewarned that all trials would consist of eight digits and were instructed to remember them while ignoring any letters that might be presented in the same trial. Additionally, during immediate written serial recall, the participants were urged to guess the identity of any digit they thought they had forgotten before proceeding to recall the next item in the sequence.

The participants initiated the onset of each trial. Prior to the experiment proper, the participants completed two practice trials from each condition. Experiment $2 \mathrm{~B}$ had the additional instruction that the participants should fixate the center of the monitor throughout each trial until cued for recall.

\section{Results and Discussion}

Experiment 2A. Responses were scored with respect to a strict serial recall criterion: An item had to be in its cor- rect position to be counted as correct (see Figure 2, top panel). The results broadly support the hypotheses advanced above: Recall was at its worst when the irrelevant sequence was both changing (which led to poor streaming but also to an irrelevant sound effect) and had the same pitch as the to-be-remembered sequence (which again militated against streaming). Increasing the pitch difference (to $\mathrm{C} 5$ ) reduced the degree of disruption, but performance was still worse than, for example, R5, where streaming was promoted both by pitch difference and by repetition of the token.

When performance in the five conditions was compared in a one-way repeated-measures ANOVA, the effect of acoustic conditions was significant $[F(4,100)=84.25$, $\left.M S_{\mathrm{e}}=1.07, p<.0001\right]$. A Tukey/Kramer comparison $(\alpha=.05$; critical difference for all comparisons $=6.4 \%)$ showed that both the R0 (64.6\% of items recalled correctly) and R5 (66.2\% of items recalled correctly) conditions were not significantly different. Further, performance in
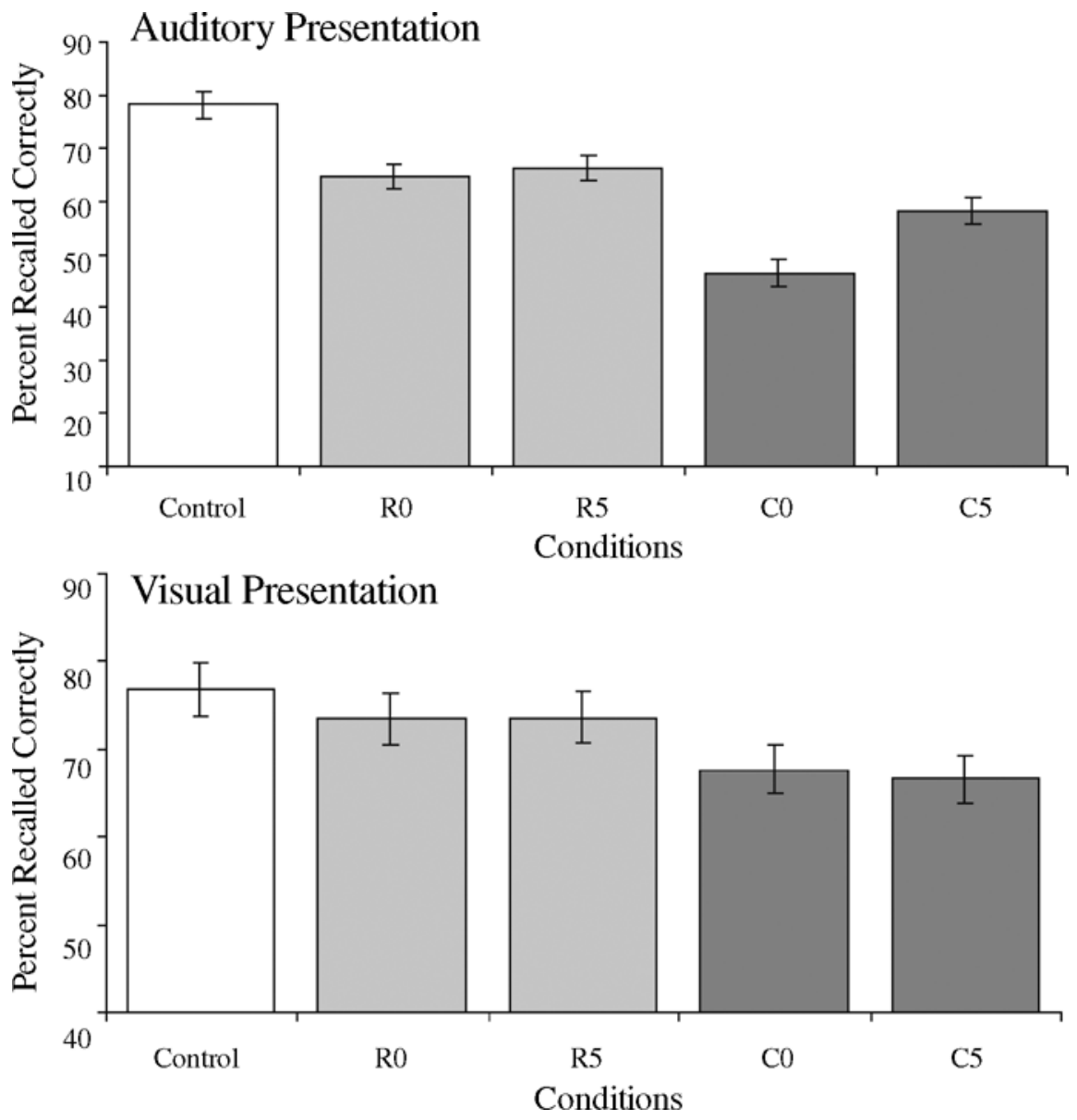

Figure 2. The effect of interpolated spoken irrelevant material on the recall of items (top panel, Experiment 2A, auditory lists; bottom panel, Experiment $2 \mathrm{~B}$, visual lists). The letters $\mathrm{R}$ and $\mathrm{C}$ denote conditions in which the irrelevant sequence consisted of a repeated or a changing token, respectively. The numeric value proceeding each letter represents the pitch (in semitones) below list-pitch (relative to Experiment $2 \mathrm{~A}$ ) at which irrelevant sequences were presented. Standard error bars are displayed. 
repeated conditions was generally better than that in changing conditions. The difference between the changing conditions was significant: A changing sequence five semitones below list-pitch, C5 (58.1\%), resulted in recall superior to that in the changing sequence presented at listpitch, $\mathrm{C} 0(46.4 \%)$.

In order to test the interplay of change within the irrelevant sequence and pitch, the control condition was excluded, and a further $2 \times 2$ repeated measures ANOVA was undertaken with the factors sequence type (repeated vs. changing) and pitch difference (0- or 5-semitone difference). All terms proved significant [effect of sequence type, $F(1,25)=79.44, M S_{\mathrm{e}}=1.46, p<.0001$; effect of pitch, $F(1,25)=34.69, M S_{\mathrm{e}}=0.85, p<.0001$; and interaction of factors, $F(1,25)=24.47, M S_{\mathrm{e}}=0.705, p<$ $.0001]$. It is clear from Figure 2 and the preceding analysis that the source of the interaction was due to the role of pitch difference in determining disruption produced by the changing, but not the repeated, conditions.

The modulation in the degree of disruption by irrelevant stimuli, evident in Experiment 2, can be predicted generally by what is already known about perceptual organization of auditory sequences.

Experiment 2B. Here again, the results were as predicted (see Figure 2, bottom panel). There was a small effect of a repeated sequence, a larger effect of a changing sequence, but no effect of pitch and no interaction between pitch and degree of change. It would be very surprising indeed if there had been an effect of pitch, given that the pitch difference relates to the auditory presentation of Experiment $2 \mathrm{~A}$.

In a one-way ANOVA, the main effect of auditory condition was significant $\left[F(4,25)=14.85, M S_{\mathrm{e}}=0.858, p<\right.$ $.0001]$. A Tukey/Kramer comparison $(\alpha=.05$; critical difference for all comparisons $=4.5 \%)$ demonstrated that both conditions in which repeated tokens $(\mathrm{R} 0=73.4 \%$; $\mathrm{R} 5=73.5 \%$ ) were presented were not significantly different from each other or from the control condition (control $=76.9 \%$ ). In contrast, the effect of a changing irrelevant sequence was marked and significant $(\mathrm{C} 0=67.7 \%$; C5 $=66.6 \%$ ). This was true of the comparison between control and each of the repeated conditions. No significant difference was found in performance between the changing conditions.

As a further check on the interaction, a repeated measures 2 (sequence type: repeated vs. changing) $\times 2$ (pitch: 0 - or 5-semitone difference) ANOVA was performed in which the control condition was excluded. There was a significant main effect of sequence type $[F(1,25)=21.67$, $\left.M S_{\mathrm{e}}=1.20, p<.0001\right]$, but no significant main effect of pitch $(F<1)$. Further, the interaction between the two effects was nonsignificant $\left[F(1,25)=0.40, M S_{\mathrm{e}}=0.77, p=\right.$ .53].

The differences in outcome between Experiments 2A and $2 \mathrm{~B}$ can be explained by the role of streaming and the irrelevant sound effect. When partitioning between events is made easy, the usual changing state effect occurs. This is seen in the contrasts $\mathrm{R} 0-\mathrm{C} 0$ and $\mathrm{R} 5-\mathrm{C} 5$ in the visual modality (Experiment 2B). Additionally, the R5-C5 contrast in the auditory modality (Experiment $2 \mathrm{~A}$ ) gives an estimate of the irrelevant sound effect that is relatively uncontaminated by the difficulty of partitioning relevant from irrelevant sequences. If we take this at face value, we arrive at an estimate of the irrelevant sound effect in the auditory modality $(\mathrm{R} 5-\mathrm{C} 5$ difference $=8.1 \%)$ that is not too dissimilar from that found in the visual modality (R5C5 difference $=6.9 \%$ ). The modality of presentation produces one other difference; however, the contrast between repeated and control conditions is larger in the auditory case than in the visual case. This may be because of some residual cost of partitioning, or it could be a sampling error between participants.

In Experiment 2B, a Cohen's $d$ of 0.36 suggests that the size of the changing state effect (the difference between $\mathrm{R} 5$ and $\mathrm{C} 5$ conditions) is small to moderate. In Experiment 2A, the size of the effect between R5 and C5 was slightly larger but still moderate (Cohen's $d=0.59$ ). In contrast, there is an extremely large effect of change in sandwich conditions ( $\mathrm{R} 0$ and $\mathrm{C} 0$ ) presented at list-pitch in Experiment 2A (Cohen's $d=1.33$ ).

\section{GENERAL DISCUSSION}

To summarize, we showed in Experiment 1 that the small effect obtained by Baddeley et al. (1993) was due to the use of a span procedure in conjunction with a low token dose. The sandwich effect was much larger than that found hitherto. Experiment 2 illustrated the manner in which the partitioning of sequences could be promoted by well-established manipulations from the auditory streaming literature: pitch differences across sequence and also similarity of token identity within sequence. These two manipulations reduced the overall effect of error in sandwich sequences, but there was also a residual error that can be ascribed to the irrelevant speech effect. Some measure of the true magnitude of the irrelevant speech effect uncontaminated by the partitioning of auditory streams can be gleaned from using the same auditory irrelevant stimuli but with visually presented to-be-remembered lists. Overall, the pattern of error was similar in the visual and auditory forms of the task, and, in particular, the difference between steady and changing state irrelevant sequences was closely similar in the visual case to the auditory case, in which streaming should have been most evident (the R5-C5 contrast).

The present experiments correct the conclusion of Baddeley et al. (1993) and show that the sandwich effect is substantial in magnitude and that the irrelevant sound effect takes the usual form by exhibiting a changing state effect. Experiment 1 suggests that the span method, with its relatively short lists, might have militated against the production of an irrelevant sound effect by having too few events in the irrelevant sequence associated with each list. The irrelevant sound effect is subject to token dose ef- 
fects-that is, as the number of irrelevant events increases so does the degree of disruption (see Bridges \& Jones, 1996; see also Jones, Alford, Bridges, Tremblay, \& Macken, 1999). In addition, more irrelevant items per unit time encourages fission, just as has been demonstrated in many studies of listening (for an overview, see Bregman, 1990; Handel, 1989; Van Noorden, 1975). This will be important when both list and irrelevant items are presented in the auditory modality. Generally, therefore, for effects of streaming to be made manifest within lists, the sequences should be long and/or should have a high dose of irrelevant items present: criteria that were again not met fully in the Baddeley et al. (1993) study.

From the standpoint of modality, it is clear from the present results that as long as the effects of alternating auditory items are minimized by adequate streaming, the irrelevant speech effect in the auditory modality will be strikingly similar to that in the visual modality. This harmonizes well with the generalization of the object-oriented episodic record (O-OER model) of short-term memory (e.g., Jones, 1993; Jones, Beaman, \& Macken, 1996) that verbal events are represented as "amodal, abstract representations... [and that] ... the origin of codes in short-term memory should not be a critical factor in determining disruption in serial recall" (Macken \& Jones, 1995, p. 437). The O-OER model regards the effect of irrelevant sound as the conflict of two processes of seriation, one from the obligatory processing of serial order of the irrelevant sound, the other from the rote rehearsal of the to-beremembered lists. In acknowledging the common representation, the model assumes that there will be structural differences between modalities so that organizational factors in vision will be different from those of audition, for example. Once these differences are minimized (as in some of the conditionsin the present experiments), the common nature of representation becomes evident.

In another paper by Baddeley and colleagues, the small magnitude of the sandwich effect was claimed to be antithetical to chaining theories of short-term retention (Baddeley, Papagno, \& Norris, 1991). By the same token, the present results showing a strong sandwich effect might now be taken as evidence in favor of chaining: The process of stream segregation could be seen as setting up two chains of sequences that in turn could be supported by chaining processes. An alternative view is that models devoid of (item-item) chaining (e.g., competitive queuing models) can incorporate the effects of streaming by having two stages, first a streaming stage, and then a competitive queuing stage. This is the case in the primacy model of Page and Norris (1998). However, although the mechanism of retrieval based on primacy gradient is described fully, it is not clear what mechanism is responsible for streaming.

In sum, the present paper helps to correct the impression given by Baddeley et al. (1993) on the size of the sandwich effect. The present results have illuminated the interplay of streaming and irrelevant sound effects by showing that both are predictable from a substantial body of precedent. At the same time, the contrast between effects in the auditory and visual modalities illustrated the functional similarity of the effect of irrelevant sound on auditory and visual lists.

\section{REFERENCES}

BADDELey, A. D. (1986). Working memory. Oxford: Oxford University Press, Clarendon Press.

Baddeley, A. D., Papagno, C., \& Andrade, J. (1993). The sandwich effect: The role of attentional factors in serial recall. Journal of Experimental Psychology: Learning, Memory, \& Cognition, 19, 862-870.

Baddeley, A. D., Papagno, C., \& Norris, D. (1991). Phonological memory and serial order: A sandwich for TODAM. In W. E. Hockley $\&$ S. Lewandowsky, (Eds.), Relating theory and data: Essays on human memory in honor of Bennnet B. Murdock (pp. 175-194). New Jersey: Erlbaum.

Bregman, A. S. (1990). Auditory scene analysis: The perceptual organization of sound. Cambridge, MA: MIT Press.

Bridges, A. M., \& Jones, D. M. (1996). Word dose and the disruption of serial recall by irrelevant speech: Phonological confusions or changing state? Quarterly Journal of Experimental Psychology, 49, 919939.

Cohen, J., MacWhinney, B., Flatt, M., \& Provost, J. (1993). PsyScope: An interactive graphic system for designing and controlling experiments in the psychology laboratory using Macintosh computers. Behavior Research Methods, Instruments, \& Computers, 25, 257-271.

Colle, H. A., \& Welsh, A. (1976). Acoustic masking in primary memory. Journal of Verbal Learning \& Verbal Behavior, 15, 17-31.

Hamilton, P., \& Hockey, R. (1974). Active selection of items to be remembered: The role of timing. Cognitive Psychology, 6, 61-83.

HANDEL, S. (1989). Listening: An introduction to the perception of auditory events. Cambridge, MA: MIT Press.

Hanley, R. J., \& Broadbent, C. (1987). The effect of unattended speech on serial recall following auditory presentation. British Journal of Psychology, 78, 287-297.

HiтcH, G. J. (1975). The role of attention in visual and auditory suffix effects. Memory \& Cognition, 3, 501-505.

JoNES, D. M. (1993). Objects, streams and threads of auditory attention. In A. Baddeley \& L. Weiskrantz (Eds.), Attention: Selection, awareness and control (pp. 87-103). Oxford: Oxford University Press, Clarendon Press.

Jones, D. M., Alford, D., Bridges, A., Tremblay, S., \& Macken, W. J. (1999). Organizational factors in selective attention: The interplay of acoustic distinctiveness and auditory streaming in the irrelevant sound effect. Journal of Experimental Psychology: Learning, Memory, \& Cognition, 25, 464-473.

Jones, D. M., Beaman, C. P., \& Macken, W. J. (1996). The objectoriented episodic record model. In S. Gathercole (Ed.), Models of shortterm memory (pp. 209-238). London: Erlbaum.

Jones, D. M., \& MACKEN, W. J. (1993). Irrelevant tones produce an irrelevant speech effect: Implications for phonological coding in working memory. Journal of Experimental Psychology: Learning, Memory, \& Cognition, 19, 369-381.

JoNES, D. M., \& MACKEN, W. J. (1995a). Auditory babble and cognitive efficiency: Role of number of voices and their location. Journal of Experimental Psychology: Applied, 1, 216-226.

Jones, D. M., \& MACKen, W. J. (1995b). Organizational factors in the effect of irrelevant speech: The role of spatial location and timing. Memory \& Cognition, 23, 192-200.

Jones, D. M., \& MACKen, W. J. (1995c). Phonological similarity in the irrelevant speech effect: Within- or between-stream similarity? Journal of Experimental Psychology: Learning, Memory, \& Cognition, 21, 103-115.

Jones, D. M., Madden, C., \& Miles, C. (1992). Privileged access by irrelevant speech to short-term memory: The role of changing state. Quarterly Journal of Experimental Psychology, 44, 645-669.

LeСомPTE, D. C. (1994). Extending the irrelevant speech effect beyond 
serial recall. Journal of Experimental Psychology: Learning, Memory, \& Cognition, 20, 1396-1408.

LECompte, D. C. (1995). An irrelevant speech effect with repeated and continuous background speech. Psychonomic Bulletin \& Review, 2, 391-397.

LECompte, D. C. (1996). Irrelevant speech, serial rehearsal and temporal distinctiveness: A new approach to the irrelevant speech effect. Journal of Experimental Psychology: Learning, Memory, \& Cognition, 22, 1154-1165.

MAcken, W. J., \& Jones, D. M. (1995). Functional characteristics of the inner voice and the inner ear: Single or double agency? Journal of Experimental Psychology: Learning, Memory, \& Cognition, 21, 436-448.

Martin-Loeches, M., \& Sommer, W. (1998). Testing models of the irrelevant speech effect in working memory with event-related potentials. Zeitschrift für Psychologie, 206, 1-22.

Miles, C., Jones, D. M., \& Madden, C. A. (1991). Locus of the irrelevant speech effect in short-term memory. Journal of Experimental Psychology: Learning, Memory, \& Cognition, 17, 578-584.

NeATH, I. (1999). Modeling the disruptive effects of irrelevant speech on order information. International Journal of Psychology, 34, 410-418.
Neath, I. (2000). Modeling the effects of irrelevant speech on memory. Psychonomic Bulletin \& Review, 7, 403-423.

Nicholls, A. P., \& Jones, D. M. (2002). Capturing the suffix: Cognitive streaming in immediate serial recall. Journal of Experimental Psychology: Learning, Memory, \& Cognition, 28, 12-28.

PAge, M. P. A., \& Norris, D. (1998). The primacy model: A new model of immediate serial recall. Psychological Review, 105, 761-781.

Penney, C. G., \& Blackwood, P. A. (1989). Recall mode and recency in immediate serial recall: Computer users beware. Bulletin of the Psychonomic Society, 27, 545-547.

SAlamé, P., \& BADDEley, A. (1982). Disruption of short-term memory by unattended speech: Implications for the structure of working memory. Journal of Verbal Learning \& Verbal Behavior, 21, 150-164.

VAN Noorden, L. P. A. S. (1975). Temporal coherence in the perception of tone sequences. Unpublished doctoral dissertation, Eindhoven University of Technology.

(Manuscript received December 19, 2000; revision accepted for publication September 1,2001.) 